\title{
Goethes Archivbegriff und Archivpoetik am Beispiel einer Szene aus Faust II
}

\section{Das Archiv als Wissensordnung}

Einer der ersten Philologen, die sich Goethes Leidenschaft des Archivierens und der Aktenführung annahmen, war Ernst Robert Curtius. ${ }^{1}$ In seinem Essay „Goethes Aktenführung“, in dem er sich u. a. auf den ersten Band der von Willy Flach besorgten Ausgabe von Goethes Amtliche[n] Schriften bezieht, lässt er den Dichter als Organisator von Schriftstücken auftreten. Da findet man die Feststellung:

Die Behandlung von Verwaltungsgeschäften bietet ihm das Modell und die Technik für die Organisation seiner persönlichen Existenz. Man erfaßt etwas von Goethes Lebensart und Lebensluft, wenn man ihn beim Anlegen und Führen vielfältigster Akten betrachtet. (Curtius 1954, 61)

Dabei sind Curtius’ Ausführungen nicht nur von anekdotischem Interesse: Indem er die Tätigkeiten des Rubrizierens, der Ablage von Schriftstücken in einzelnen Fächern sowie die periodischen Löschungsaktionen, die in den Tagebüchern mit zunehmender Häufigkeit vermerkt werden, in den Blick nimmt, bringt er die Einsicht ans Licht, dass Goethes Wissen von der Welt sich keinesfalls nur seiner vielberufenen Vorliebe für die Anschauung verdankt. Curtius spürt demgegenüber mit merklichem Vergnügen die Stellen in Goethes Werk auf, an denen die sichtbare Welt der Natur der Ordnung des Archivs einverleibt wird. So, als Goethe in

1 Vgl. aber auch Flach 1956. In jüngerer Zeit hat sich Bernhard J. Dotzler zu Goethes Aktenführung geäußert, vgl. Dotzler 2002. Er versteht Goethes Aktenführung als Medium, durch dessen perfekte, aber verschwiegene Beherrschung Goethe die Organisation von Wissen und die „Bewältigung (zu) großer Datenmengen“ (Dotzler 2002, 11) gelingen kann. Nicht mit Goethes Archiven, sondern mit seinen Sammlungen beschäftigten sich dagegen Trunz 1980 und Asman 1997. Die Dokumentation in der Sammlung unterscheidet sich von der im Archiv durch ihre Gegenständlichkeit: Das Archiv dient Goethe als Zwischenlager für die Weiterbearbeitung von Schriftstücken (in der eigenen schriftstellerischen Tätigkeit ebenso wie im Austausch mit anderen) und nicht von Anschauungsgegenständen. Das Archiv operiert im Unanschaulichen. Beide haben allerdings gemeinsam, dass die gespeicherten Materialien vor allem für Zwecke der Kommunikation aufbewahrt werden und dass daher die Untersuchung ihres Aufbaus Aufschlüsse über Wissensordnungen zulässt. Vgl. dazu am Beispiel der Kunstkammer Bredekamp 1993 und zur Entwicklung von Archiven Pompe und Scholz 2002. Eine interessante Studie zum Archiv bei Goethe ist Bez 2013. 
einem Brief vom 25. September 1797 Schiller aus der Schweiz mit dem Enthusiasmus eines Sammlers mitteilt, „die Rubrik dieser ungeheuern Felsen“ - gemeint sind die Berge des Vierwaldstätter Sees - „darf mir unter meinen Reise-Kapiteln nicht fehlen“ (FA 11/4, 431). Die Natur, so bemerkt Curtius dazu, werde „ad acta genommen“ (Curtius 1954, 65). Die Aktenführung hat für Goethe demnach eine epistemologische Bedeutung, sie ist für ihn nicht nur ein Hilfsmittel, um seine Schriftstücke zu organisieren, sondern auch ein Denkmodell, mit dem er die Wirklichkeit betrachtet. Daher fungiert das Archiv mit seiner Fachterminologie in Goethes Sprachgebrauch als Metaphernfeld, das er verwendet, um Gesehenes, Gehörtes, Gelesenes zu bewerten und mental zu sortieren. Nicht zuletzt prägen Archivpraxis und Archivsemantik, wie in der germanistischen Forschung mehrfach gezeigt wurde, Goethes Schreiben, vor allem seine späten Werke Wilhelm Meisters Wanderjahre und Faust II. Im Folgenden möchte ich zunächst Goethes Archivverständnis kurz skizzieren und dann an einer ausgewählten Stelle aus Faust II zeigen, wie das Drama zu einem Archiv werden kann.

Goethes Archivbegriff unterscheidet sich vom üblichen Sprachgebrauch seiner Zeit. Das Wort Archiv ist im 18. Jahrhundert nämlich ausschließlich der staatlichen Institution des Archivs vorbehalten. ${ }^{2}$ Demgegenüber verwendet Goethe das Wort in einem erweiterten Sinn, um damit seine private Sammlung verschiedenartiger Schriftstücke und deren Organisation zu bezeichnen. Diese Übertragung des Wortes aus dem Bereich staatlicher Macht auf den Privatgebrauch des Schriftstellers hat ihren Grund zunächst einmal in der vergleichbaren Aufgabe beider Archive. Wie unterschiedlich die darin aufbewahrten Dokumente und die Regeln für die Benutzung auch sein mögen, so teilen sie dennoch ihre zentrale Funktion der Datenspeicherung. Das hier Abgelegte soll für zukünftige Kommunikation verfügbar sein, damit aus den Daten wiederum Information werden kann. Doch trotz dieser Gemeinsamkeit sind die Unterschiede zwischen staatlichem und Privatarchiv gravierend. Sie betreffen die Art und Verwendung der Archiveinträge, die Regeln für die Nutzung, die rechtliche Situation und die Ordnung und Pflege des Archivs.

2 Hierzu Flach: „Durch hundert Jahre hindurch, von Zedler bis hin zu Rotteck und Welcker, im Jahrhundert Goethes also, ist demnach die allgemeine Auffassung vom Wesen des Archivs als einer territorialstaatlichen Angelegenheit im Grunde die gleiche geblieben“ (Flach 1954, 47). Und er konstatiert mit Bezug auf Goethes Sprachgebrauch, dass „,er über den landläufigen Begriff des Archivs als einer staatlichen Einrichtung hinaus für dieses Wort bereits sehr viel weitergehende Deutungen und Auffassungen hat, so weitgehende Deutungen, daß der Ausgangspunkt mitunter völlig verlassen wird“ (Flach 1954, 60). Über den goethezeitlichen Archivbegriff informiert auch Bez 2013, 22. 
Was die Ordnung von Goethes Privatarchiv betrifft, so ist diese recht traditionell angelegt. Das grundlegende Ordnungsprinzip bildet nämlich die Rubrik: Einige dieser Rubriken heißen zum Beispiel ,Publica‘ und ,Privata‘, ,Domestica“ und ,Religiosa'. Damit etabliert Goethe eine semantische Ordnung, die ihn dazu zwingt, jedes Dokument einem bereits vorgefertigten Topos zuzuordnen, der auf eine in der Wirklichkeit vorausgesetzte Sphäre rekurriert. Goethe folgt damit der durch die Rhetorik und das Medium des Buchs geprägten Praxis der Wissensverwaltung, die sich erst im Verlauf des 18. Jahrhunderts langsam verabschiedet. ${ }^{3}$ Der Nachteil daran ist, dass bei jedem Dokument entschieden werden muss, zu welchem Bereich es gehört, was keine große Flexibilität erlaubt. Eine Alternative dazu wäre eine semantisch leer numerische Ordnung, die lediglich die Stelle eines Dokuments im Archiv bezeichnet und sich von der Referenz auf Wirklichkeit befreit - damit dann aber vielfältige Referentialisierungen auf unterschiedliche Gebiete ermöglichen würde. Soll dagegen in Goethes Archiv ein unter ,Privata“ abgelegtes Dokument für neue Zwecke genutzt werden, so muss er stets wissen, unter welcher Rubrik er es auffinden kann. Es ist also kein Wunder, wenn das Archiv im Lauf der Zeit unüberschaubar und damit dysfunktional wird. Goethe entschloss sich erst im Alter dazu, den notwendigen Schritt einer gründlichen Neuordnung zu unternehmen. Hierüber berichtet er in einem Aufsatz mit dem Titel Archiv des Dichters und Schriftstellers, den er 1823 im ersten Heft des vierten Bands von Über Kunst und Altertum veröffentlichte, und zwar - dies verdient besondere Beachtung - zwischen zwei autobiografischen Texten mit den Titeln Selbstbiographie und Lebensbekenntnisse im Ganzen. Durch diese Publikationsweise verdeutlicht er die Bedeutung des Archivs für die narrative Vergegenwärtigung und Deutung seines Lebens.

Gegenstand der Notiz über das eigene Archiv ist die von Goethes Sekretär Kräuter unternommene Arbeit, ein Repertorium (Findbuch) herzustellen. Für die Neuordnung des Archivs gab es zwei konkrete Anlässe, nämlich erstens Goethes Vorhaben, seine Lebenserinnerungen zu bearbeiten, die 1830 als Tag- und JahresHefte erschienen, und zweitens die Herausgabe der Werke letzter Hand, die er 1822 in Angriff nahm. ${ }^{4}$ Über Kräuters Arbeit heißt es nun:

Ein junger, frischer, in Bibliotheks- und Archivgeschäften wohlbewanderter Mann (Bibliothekssekretär Kräuter) hat es diesen Sommer über dergestalt geleistet, daß nicht allein

3 Vgl. Zedelmaier 2002, 48: „Erst allmählich verlor die private Wissensvorsorge ihre Gedächtnisund Buchzentriertheit. Der eigentliche Umbau der ortsgebundenen, topischen Wissensverwaltung zu pragmatischen, flexibel erweiterbaren Zettelkästen setzt erst im 18. Jahrhundert ein.“

4 Über die Neuordnung des Archivs unterrichtet ausführlich Flach 1965, 60-62; über das Repertorium bes. 66-68. Auch zu den Aufsätzen finden sich auf S. 65 einige Anmerkungen. 
Gedrucktes und Ungedrucktes, Gesammeltes und Zerstreutes vollkommen beisammensteht, sondern auch die Tagebücher, eingegangene und abgesendete Briefe in einem Archiv beschlossen sind, worüber nicht weniger ein Verzeichnis, nach allgemeinen und besondern Rubriken, Buchstaben und Nummern aller Art gefertigt, vor mir liegt [...]. (GA 12, 650)

Über die erwähnten konkreten Anlässe der Neuordnung des Archivs hinaus zeigt das Triptychon der Aufsätze, dass Goethe eine Verbindung zwischen dem Archiv in seiner Materialität und der Deutung der Existenz erkennt. Der Abschnitt mit dem Titel Selbstbiographie befasst sich mit der Frage, wie die Darstellung der Vergangenheit gelingen könne. Das Problem besteht in dem unweigerlichen Verblassen oder Verschwinden der Erinnerung, die zu einem späteren Zeitpunkt nicht mehr ohne Weiteres wiederbelebt werden kann:

Es ist keine Frage, daß uns die Fülle der Erinnerung, womit wir jene ersten Zeiten zu betrachten haben, nach und nach erlischt, daß die anmutige Sinnlichkeit verschwindet und ein gebildeter Verstand durch seine Deutlichkeit jene Anmut nicht ersetzen kann. (GA 12, 648)

Aus der Perspektive des gealterten Menschen steht der Zusammenhang dieses Ganzen nämlich nicht mehr fest, vielmehr „kommt [man] zuletzt beinahe in den Fall, wie jener Geometer nach Endigung eines Theaterstücks auszurufen: was soll denn das aber beweisen?“ (GA 12, 648). Die Kunst der Erinnerung besteht demzufolge darin, von dieser resignativen Erinnerung abzurücken und sich wieder jenes Gefühl des Anfangs ins Gedächtnis zu rufen, als alles noch danach aussah, als könne die Kohärenz des Lebens erreicht werden. Es komme darauf an, heißt es im Text, „sich wieder dahin [zu] stellen, wo man noch hofft, ein Mangel lasse sich ausfüllen, Fehler vermeiden, Übereilung sei zu bändigen und Versäumtes nachzuholen“. Der Übergang von dieser Reflexion auf die Mitteilungen über das Archiv wird in einem letzten Abschnitt vollzogen: Die Überarbeitung und Neuorganisation des Archivs sowie die Anlage des Findbuchs sind ausdrücklich Hilfsmittel, um sich aus der „hypochondrischen Ansicht“, es könne dem Leben keine Ganzheit verliehen werden, „zu retten“ (GA 12, 648).

Das Archiv als Rettung - Rettung aus dem Vergessen, dem Gefühl der Inkohärenz, der Unvollständigkeit des eigenen Lebens. Die Souveränität des Subjekts hängt nicht zuletzt ab von den Lebensspuren, die in die Materialität des Archivs eingetragen wurden und die zu einem späteren Zeitpunkt wieder abrufbar sein müssen. Behandelt der erste der drei Aufsätze also die Schwierigkeiten einer narrativen Rekonstruktion der Lebensgeschichte und der mittlere deren Basis, das Archiv, so kann der dritte Teil mit einer optimistischen Aussage die Vorteile eines geordneten und funktionierenden Privatarchivs auf den Punkt bringen: 
Nun liegen nicht alleine diese [die Tagebücher], sondern so viele andere Dokumente nach vollbrachter archivarischer Ordnung aufs klarste vor Augen und ich finde mich gereizt jenen Auszug aus meiner ganzen Lebensgeschichte dergestalt auszuarbeiten, daß er das Verlangen meiner Freunde vorläufig befriedigte und den Wunsch nach fernerer Ausführung wenigstens gewisser Teile lebhaft errege: woraus denn der Vorteil entspringt, daß ich die gerade jedesmal mir zusagende Epoche vollständig bearbeiten kann und der Leser doch einen Faden hat, woran er sich durch die Lücken folgerecht durchhelfen möge. (GA 12, 651)

In diesem Zitat deutet Goethe eine Besonderheit des Archivs an, die er auch in anderen Zusammenhängen gerne unterstreicht: Es ist seine eigentümliche Zeitlichkeit, die quer steht zu der Zeitlichkeit des gelebten Lebens. Denn das Leben bewegt sich vorwärts und auch die Erinnerung an die Vergangenheit bleibt immer eine Erinnerung in der Gegenwart, also abhängig von vielfältigen Faktoren Emotionen, Stimmungen, äußeren und inneren Gegebenheiten -, während das Archiv aus der Komplexität des Lebendigen nur dürre Spuren konserviert. Die Zeit wird im Archiv in Raum umgewandelt: den Raum der Dinge, der Dokumente, der Schrift, die erstarren und keine Bedeutung mehr besitzen, solange sie nicht von einem Benutzer betrachtet und mit einem stets kontingenten, weil vom jeweiligen Interesse des Betrachters abhängigen Sinn aufgeladen werden. Gerade diese Zeitlosigkeit des Archivs ermöglicht aber die Dynamik seiner Verwendungen. So kann der gealterte Autor frei bestimmen, welche Periode seines Lebens er gerade erzählen möchte, weil sein Archiv geordnet funktioniert und ihm jederzeit den Zugriff auf die Daten ermöglicht.

Das erklärt, warum für Goethe das Archiv eine so zentrale Werkstätte ist: Ein Autor wie er lebt nicht aus seinem subjektiven Empfinden, sondern aus dem, was er aus Wissen, Erfahrung, Erkenntnis, Neugierde, Sensibilität und Fantasie sich angeeignet, angelesen und erworben hat. Die Archivierung dieses Erworbenen ermöglicht ihm eine gewisse Distanzierung und Freiheit und Souveränität gegenüber der Wirklichkeit und, was vielleicht noch wichtiger ist, gegenüber den Meinungen und Interpretationen, die unablässig von dieser Wirklichkeit erzeugt werden. Diese Rolle des Archivs hebt Goethe ganz besonders anlässlich seiner geplanten zweiten Reise nach Italien im Jahr 1797 hervor. Geplant war ursprünglich eine enzyklopädische Reise, während der Goethe anhand vorgefertigter Rubriken zahlreiche empirische Daten in Italien erheben und zu einem Gesamtbild des dortigen Lebens zusammenfügen wollte. Jedoch scheitert die Reise wegen Napoleons Italienfeldzug, und Goethe besucht stattdessen die Schweiz. Die vorgefertigten Rubriken, mit denen er Italien ,ad acta' nehmen wollte, werden nun an anderen Gegenständen erprobt, und zwar an den vielfältigsten Gegenständen, denn Goethe hält Ausblick nach Stoffen für zukünftige Werke, Steinen für seine Sammlungen, Anregungen für seine Intendantentätigkeit und vieles mehr. An Schiller berichtet er am 25. September: 
Ich habe schon ein paar tüchtige Aktenfaszikel gesammelt, in die alles, was ich erfahren habe, oder was mir sonst vorgekommen ist, sich eingeschrieben oder eingeheftet befindet, bis jetzt noch der bunteste Stoff von der Welt, aus dem ich auch nicht einmal, wie ich früher hoffte, etwas für die Horen herausheben könnte. (FA I/4, 431)

Hier zeigt sich das Archiv gewissermaßen von seiner anderen Seite: Während in den späteren Reflexionen des alten Goethe die Verwertung des Archivierten im Zentrum steht, geht es bei der dritten Schweizer Reise um das Sammeln von Archivmaterialien. Obwohl diese Tätigkeit von Anfang an bereits die zukünftige Verwendung einplant, werden die Materialien keiner vorgängigen Triage unterzogen, denn Goethe betont ausdrücklich deren Buntheit. In dieser nicht hierarchischen Tätigkeit unterscheidet sich der Privatarchivar Goethe natürlich von seinem ernsten Kollegen im staatlichen Archiv, der sich eine solche Sorglosigkeit nicht erlauben kann und wohl auch nicht unbedingt erlauben will.

Das Archivieren und Sammeln ermöglicht es dem Reisenden Goethe, die Informationen der Wirklichkeit nicht sogleich beurteilen, interpretieren, bewerten oder verwenden zu müssen. Der Zustrom an Neuem wird am Urteilsvermögen vorbei ins Archiv gelenkt und als Vorrat für spätere Verwendung angelegt, auch wenn noch keinesfalls klar ist, worin diese bestehen könnte. Gegenüber Schiller beschreibt er das so:

Ich gewöhne mich nun alles wie mir die Gegenstände vorkommen und was ich über sie denke aufzuschreiben, ohne die genaueste Beobachtung und das reifste Urteil von mir zu fordern, oder auch an einen zukünftigen Gebrauch zu denken. Wenn man den Weg einmal ganz zurückgelegt hat so kann man mit besserer Übersicht das vorrätige immer wieder als Stoff gebrauchen. (FA I/4, 431)

Aufgrund seiner besonderen Zeitlichkeit ermöglicht das Archiv also eine Urteilsenthaltung, während es das Eingetragene zugleich für eine spätere Beurteilung bzw. Verwertung bereithält. Es gibt aber auch den entgegengesetzten Fall, dass Goethe auf die Archivmetaphorik zurückgreift, um bestimmte Standpunkte oder Meinungen zu verwerfen, wie aus einer Äußerung über Alexander von Humboldts Fragments de Géologie hervorgeht - eine Schrift, in der der große Naturforscher die Entstehung des Himalajas als Folge vulkanischer Tätigkeit erklärte. Hierzu schreibt Goethe am 5. Oktober 1831 an Carl Friedrich Zelter:

Wenige Menschen sind fähig, überzeugt zu werden; überreden lassen sich die meisten, und so sind die Abhandlungen die uns hier vorgelegt werden wahrhafte Reden, mit großer Fazilität vorgetragen, so daß man sich zuletzt einbilden möchte, man begreife das Unmögliche. Daß sich die Himalaja-Gebirge auf $25000^{\prime}$ aus dem Boden gehoben und doch so starr und stolz als wäre nichts geschehen in den Himmel ragen, steht außer den Grenzen meines Kopfes, in den düstern Regionen, wo die Transsubstantiation pp. hauset, und mein Zerebralsystem müßte ganz umorganisiert werden - was doch schade wäre - wenn sich Räume für 
diese Wunder finden sollten. Nun aber gibt es doch Geister, die zu solchen Glaubensartikeln Fächer haben, neben sonst ganz vernünftigen Lokulamenten; ich begreif es nicht, vernehm es aber doch alle Tage. Muß man denn alles begreifen? Ich wiederhole: unser Welteroberer ist vielleicht der größte Redekünstler. (FA II/11, 473-474)

Goethe vergleicht hier sein „Zerebralsystem“ mit einem Archiv, das durch die Wörter „Fächer“ und „Lokulament“ indirekt bezeichnet wird; die Archivmetaphorik dient ihm dazu, zwischen sinnvollen und absolut sinnlosen Archiveinträgen zu unterscheiden, Wissenswertes von Unnützem zu trennen.

Goethes Archivbegriff ist also, alles in allem, schillernd und umfassend: Er bezeichnet ganz konkret das Privatarchiv des Schriftstellers, der das ihn Faszinierende vorbehaltlos sammelt, um es zu einem späteren Zeitpunkt zu betrachten und zu verwenden; es garantiert ihm die Möglichkeit, im rückblickenden Erzählen sein Leben als eine Ganzheit zu begreifen, und bildet in seiner Materialität die Basis für Erinnerung und Selbstreflexion. Zugleich dient es aber auch als Metapher, um zwischen sinnvollen und nicht sinnvollen Vorstellungen und Ideen zu unterscheiden. In all diesen Fällen stiftet das Archiv eine enge Verbindung zwischen der Pluralität der Meinungen, Ansichten, Vorstellungen und ihrer materiellen Einschreibung, Speicherung, Archivierung. Goethes Archivbegriff bezweckt weniger die Verstetigung und Konservierung von Zeichen, sondern vielmehr ihre Wiederverwertung für die Zwecke der sozialen, gelehrten und nicht zuletzt literarischen Kommunikation. Sein Telos ist also nicht die Stiftung von Memoria, sondern die Metamorphose, die kreative Modifikation - kurz: die Archivpoetik.

\section{Die Archivpoetik im Faust II}

Was nun diese Archivpoetik betrifft, so meint der Begriff etwas anderes als der etablierte Terminus der ,Intertextualität‘. Zwar hat das Archiv etwas mit einem weiten Intertextualitätsbegriff zu tun, denn schließlich besteht das Archiv aus Texten und ein archivpoetischer Text wird immer auf dieses Archiv verweisen, also einen höheren oder geringeren Intertextualitätsgrad besitzen. Jedoch verbinden sich mit dem Archiv noch andere Aspekte, die ihm gegenüber der Intertextualität ein eigenes Profil verleihen. Zunächst besitzt das Archiv eine räumliche Ordnung, seine Fächer und die darin abgelegten Archivgüter müssen auffindbar sein. Ein archivpoetischer Text wird daher auch Spuren archivarischer Ordnungsverfahren enthalten. Wir haben gesehen, dass Goethes Privatarchiv lange Zeit nach dem rhetorischen Prinzip der Topik organisiert war, und es ist leicht zu zeigen, dass das Archiv Faust II demselben Prinzip gehorcht. Das Abschreiten der Handlungsorte führt Faust und seine Gefährten durch Topoi der europäischen Kultur- und Wissensgeschichte und konfrontiert ihn, durch die dramatische Rede 
der Figuren, mit dort abgelegten Archivalien bzw. mit Anspielungen auf diese, die dann von den Rezipienten und Rezipientinnen des Dramas aufgespürt und dekodiert werden können. Der Effekt davon ist zweifellos aus rezeptionsästhetischer Sicht mit dem der Intertextualität mehr oder weniger ident, aber das Textarchiv hat eine eigene Struktur. Darüber hinaus verbindet Goethe mit dem Archivbegriff einen klaren Bezug nicht einfach auf Texte im Allgemeinen, sondern auf Wissen bzw. - wie das auf Alexander von Humboldt bezügliche Zitat verdeutlicht - auf mentale Dispositionen oder, um einen Begriff Goethes zu übernehmen, auf ,Vorstellungsarten ${ }^{5}$, d. h. es geht ihm darum, das hinter dem Text liegende Archiv der Kultur zu analysieren, Denkweisen und Glaubenssysteme zu dramatisieren. Faust II, dieses gewaltige Archiv mit seiner Fülle an literarischen Anspielungen, zielt auf die Darstellung und spielerisch-poetische Analyse von Wissensformationen ab und geht damit über bloße intertextuelle Referenzen weit hinaus. ${ }^{6}$ Goethes Archiv wird zu einer Beobachtung zeitgenössischer Wissensproduktion, was ich nun abschließend an einem Beispiel aus der ,Klassischen Walpurgisnacht“ aufzeigen möchte.

Eine der großen wissenschaftlichen Debatten seiner Zeit, für die sich Goethe rege interessierte, war die Auseinandersetzung über die Entstehung der Gebirge. Es gibt um 1800 im Wesentlichen zwei Schulen der Erklärung, die sich feindlich gegenüberstehen: die neptunistische Lehre, vertreten durch Abraham Gottlob Werner, und die vulkanistische Lehre, vertreten durch Werners Schüler Johann Carl Wilhelm Voigt. Ausgangspunkt war die Deutung des Basalts, der nach Ansicht Werners ein Produkt der Kristallisation von Sedimenten aus einem hypothetischen Urozean darstellte, von Voigt dagegen richtig als Vulkangestein erkannt wurde. ${ }^{7}$ Der Neptunismus erklärt also die Bildung von Gebirgen als Folge der Sedimentation von Mineralien in dem Urozean und aus deren Kristallisation

\footnotetext{
5 Der Begriff der Vorstellungsart bezeichnet die Weise, mit der ein Mensch die Erfahrungen und empirischen Daten zu einer individuell besonderen Anschauung verknüpft, vgl. Schneider 2005, 138-145. Siehe auch Gaier 1994, 158-171.

6 Ulrich Gaier spricht aus diesem Grund von verschiedenen „Lesarten“ des Dramas, worunter er einen ,thematische[n] und gewissermaßen mitteilende[n] argumentative[n] Zusammenhang des ganzen Textes“ (Gaier 1999, 12) versteht. Für Gaier verbinden sich einzelne Elemente des Textes zu einer zusammenhängenden Aussage über Gegenstände des Wissens - etwa Religion, Naturphilosophie, Magie, Geschichte, Soziologie, Ökonomie, Anthropologie, Poetik.

7 Der Gegensatz des geologischen Vulkanismus und Neptunismus ist allerdings schon erheblich älter. So waren bereits in der Antike vulkanistische Phänomene bekannt, und es gab schon vor Werner neptunistische Theorien. Vgl. die bei Hölder 1960 versammelten Dokumente. Zum deutschen Kontext s. Wagenbreth 1999; dort findet sich auf S. 32 auch ein grafisches Schema der Werner'schen Gebirgsbildungstheorie.
} 
zu Gesteinen. Auf diese Weise kommt die neptunistische Theorie in ihrer erdgeschichtlichen Erklärung ohne Sprünge und Revolutionen aus. Letztlich zeigten Werners Schüler, zu denen neben Voigt auch Alexander von Humboldt und Leopold von Buch gehörten, aber die Bedeutung vulkanischer Aktivitäten für die Bildung von Gesteinen und Gebirgen auf und verdrängten die neptunistische Auffassung ihres Lehrers. Goethe interessierten an dieser geologischen Spezialdebatte ihre Implikationen für das Verständnis von Zeit und natürlicher Entwicklung. So versuchte er, durch eigene Thesen zum Granit, in den Streit einzugreifen und behauptete, dass der Granit ein Urgestein darstelle, das allen weiteren Entwicklungen, auch den vulkanischen, zugrunde liege. Goethe leugnet also nicht vollständig das Wirken der Vulkane, sondern nur ihren grundlegenden Einfluss auf die Erdgeschichte. Während die vulkanischen Eruptionen ereignishaft und sprunghaft sind, vollziehen sich die neptunischen Prozesse der Gesteinsbildung für Goethe in immer gleichen Zyklen. Der neptunistische Zeitbegriff ist nicht geschichtlich, sondern genetisch, regelhaft, zyklisch; der vulkanistische dagegen historisch, Ereignisse produzierend, die nicht nur in die ruhigen Naturvorgänge eingreifen, sondern auch die menschliche Geschichte beeinflussen. ${ }^{8}$

Goethe war also, was diesen wissenschaftlichen Streit angeht, höchst parteiisch. In Faust II dagegen betrachtet er das Thema, zumindest in der ,Klassischen Walpurgisnacht' mit humoristischer Distanz. Dort kommt es nämlich zu einem Erdbeben und in dessen Folge zur Entstehung eines Berges, der von dem ,in der Tiefe brummend und polternd“ (V. 7518) arbeitenden Erdgott Seismos in die Höhe gestemmt wird:

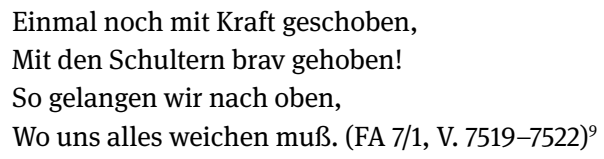

Seismos bzw. der von ihm emporgehobene Berg erscheint im Archiv der ,Klassischen Walpurgisnacht' als eine Rubrik, die auf das Fach ,Neptunisten und Vulkanisten' hinweist und die intertextuellen Referenzen der Stelle als theatrales Spiel mit der geologischen Fachdiskussion der Zeit $\mathrm{zu}$ erkennen gibt. Seismos, der offensichtlich die von den Vulkanisten beschworene erdgeschichtliche Urkraft verkörpert, wird von den anwesenden Sphingen verspottet:

8 Für den Zusammenhang von Gesteinsbildung und Zeitvorstellung bei Goethe ist interessant Pörksen 1998.

9 Faust wird im Folgenden nach der Frankfurter Ausgabe mit Versangabe zitiert. 
Er, mit Streben, Drängen, Drücken, Arme straff, gekrümmt den Rücken, Wie ein Atlas an Gebärde, Hebt er Boden, Rasen, Erde, Kies und Grieß und Sand und Letten, Unsres Ufers stille Betten. So zerreißt er eine Strecke Quer des Tales ruhige Decke. Angestrengtest, nimmer müde, Kolossale Karyatide; Trägt ein furchtbar Steingerüste, Noch im Boden bis zur Büste; Weiter aber soll's nicht kommen, Sphinxe haben Platz genommen. (V. 7536-7549)

In ihrer Verspottung des Seismos vermischen die Sphingen geologisches Fachvokabular („Kies und Grieß und Sand und Letten“) ${ }^{10}$ mit mythologischen und architektursprachlichen („Karyatide“) Elementen. Sie lassen die von Seismos behauptete Dynamik der Bergentstehung sprachlich gewissermaßen erstarren; denn alles was er, der sich anmaßt ein „Atlas“ zu sein, letztlich emporhebt, ist nur Rasen, Erde, feines Geröll. Auch wird aus dem Gott letztlich eine „Karyatide“ - ein Wort, das darauf abzielt, den männlichen Kraftanspruch zu belächeln, handelt es sich bei diesem Architekturelement doch meistens um mädchenhafte Gestalten, die nur zum Schein die erheblichen Gebäudelasten zu tragen vorgeben. Doch Seismos lässt sich nicht beirren und behauptet ein weiteres Mal, aber deutlicher noch als zuvor, einen erheblichen Anteil an der Gestaltung der Erdoberfläche zu haben.

Als, angesichts der höchsten Ahnen, Der Nacht, des Chaos, ich mich stark betrug Und, in Gesellschaft von Titanen, Mit Pelion und Ossa als mit Ballen schlug. Wir tollten fort in jugendlicher Hitze, Bis überdrüssig, noch zuletzt, Wir dem Parnaß, als eine Doppelmütze, Die beiden Berge frevelnd aufgesetzt... Apollen hält ein froh Verweilen Dort nun mit seliger Musen Chor. Selbst Jupitern und seinen Donnerkeilen Hob ich den Sessel hoch empor.

10 Laut dem Kommentar von Schöne bezeichnen diese Wörter zunehmend feiner werdende Zustände von Erde (vgl. FA I/7/2, 549). 
Jetzt so, mit ungeheurem Streben,

Drang aus dem Abgrund ich herauf

Und fordre laut, zu neuem Leben,

Mir fröhliche Bewohner auf. (V. 7558-7573)

Seismos repräsentiert demnach das vulkanistische Denken mit seinen verschiedenen Implikationen: Betonung des Ereignisses, des Revolutionären, des Sprungs. Demgegenüber stehen die Sphingen für das neptunistische Urgestein, das nur blasiert auf den polternden Halbgott herunterschauen kann:

Uralt müßte man gestehen

Sei das hier Emporgebürgte,

Hätten wir nicht selbst gesehen,

Wie sich's aus dem Boden würgte. (V. 7574-7577)

Die Antwort der Sphingen zielt darauf ab, das vermeintlich uralte seismische Wirken als in Wahrheit überaus rezent zu deklassieren. Sie heben auch hervor, dass vom Aussehen des Berges kein direkter Rückschluss auf sein Alter möglich ist, weil der Augenschein trügt: Der gerade erst entstandene Berg sieht jetzt schon so aus, als sei er uralt. Liegt dann nicht der Verdacht nahe, dass auch die anderen Berge seismischen Ursprungs bedeutend jünger sind, als es den Anschein hat? Wie auch immer man es sehen will: Das Wortgefecht erlaubt in keiner Weise eine sichere Antwort auf die Frage, wer von beiden Recht hat, Seismos oder die Sphingen. Es überträgt die erdgeschichtliche Diskussion in den dramatischen Text, aber nicht, um ein gelehrtes Drama zu erzeugen, sondern um das Drama der Gelehrsamkeit zu unterstreichen: unabhängig von der Frage, inwiefern die Wissenschaft in der Lage ist, zu Wahrheiten zu finden, sieht es so aus, als sei die Macht des Meinens nicht zu überwinden. Das wird bestätigt, als wenig später die beiden Philosophen Anaxagoras und Thales erscheinen, erneut Rubriken, mit denen sich in der Philosophiegeschichte die Erinnerung daran verbindet, dass der eine (Anaxagoras) das Feuer, der andere (Thales) das Wasser zu dem Grundprinzip des Seins erhoben hat. Folgerichtig erscheint der Feuerphilosoph Anaxagoras als Fürsprecher des Seismos, der Wasserphilosoph Thales dagegen als dessen Gegner. Und so entbrennt zwischen beiden ein hitziges Streitgespräch, bei dem sich allerdings rasch herausstellt, dass beide gar nicht über dasselbe sprechen. Denn während Anaxagoras den Ursprung des Bergs aus dem Feuer erklären möchte, geht es Thales allgemeiner um die Entstehung des Lebendigen. Anaxagoras bezieht sich daher auf den konkreten Gegenstand des Berges, der ihm und Thales unmittelbar vor Augen steht: „Dein starrer Sinn will sich nicht beugen, / Bedarf es weit’res dich zu überzeugen?“(V. 7851-7852). In seiner Antwort weicht Thales aus. Er lässt sich nicht auf das Argument des Gegners ein, sondern wechselt die Ebene: 
„Die Welle beugt sich jedem Winde gern, / Doch hält sie sich vom schroffen Felsen fern“ (V. 7853-7854). - Das ist eine allgemeine Sentenz, die sich auf die Gesprächssituation eher als auf das Naturproblem bezieht. Indem er Anaxagoras als „schroffen Felsen“ charakterisiert, bekundet Thales ausdrücklich, dass er keine Lust hat, sich mit der Meinung des Konkurrenten auseinanderzusetzen. Er wirft Anaxagoras eine Schroffheit und Unbeweglichkeit vor, dieihn in diesem Gespräch aber vor allem selbst charakterisiert, da er sich in keiner Weise auf die Voraussetzungen des Gegners einlassen mag. ${ }^{11}$ Während Anaxagoras auf seinem Thema beharrt - „Durch Feuerdunst ist dieser Fels zu Handen“ (V. 7855) -, lässt sich Thales gar nicht darauf ein: „Im Feuchten ist Lebendiges entstanden“ (V. 7856). Er verbleibt im Bereich bloßer Dogmatik, da er sich nicht auf den empirischen Augenschein einlassen will. Anaxagoras hingegen beharrt auf dem Einzelfall:

\section{THALES}

Nie war Natur und ihr lebendiges Fließen

Auf Tag und Nacht und Stunden angewiesen;

Sie bildet regelnd jegliche Gestalt,

Und selbst im Großen ist es nicht Gewalt.

ANAXAGORAS

Hier aber war's! Plutonisch grimmig Feuer,

Äolischer Dünste Knallkraft ungeheuer,

Durchbrach des flachen Bodens alte Kruste

Daß neu ein Berg sogleich entstehen mußte. (V. 7861-7868)

Wie sehr sich Thales weigert, auf das empirische Geschehen einzugehen, wird ein weiteres Mal bei dem Meteoriteneinschlag deutlich, der die Existenz gewaltsamer Kräfte in der Natur sozusagen schlagend belegt. Während die ,überhitzte‘ Imagination des Feuerphilosophen gleich den Mond vom Himmel stürzen sieht (vgl. V. 7910-7929), wo es sich ,nur' um einen Meteoriten handelt, gibt Thales vor, nichts gesehen zu haben: „Was dieser Mann nicht alles hört' und sah! / Ich weiß nicht recht wie uns geschah" (7930-7931). Und selbst als Homunkulus ihn auf die Folgen des Einschlags aufmerksam macht - (vgl. V. 7936-7937 „Schaut hin nach der Pygmäen Sitz, / Der Berg war rund, jetzt ist er spitz.“) -, erkennt er weder Anaxagoras noch die vulkanisch-plutonischen Kräfte an und entgegnet auf die

11 So auch Thomas Zabka, der zu Recht betont, dass die Haltung des Thales nicht gewaltfrei ist, weil sie den Fakten des Anaxagoras die Anerkennung verweigert: „Die neptunistischen Figuren behaupten, daß in der Wahrnehmung der Gegenpartei allein die subjektive Vorstellung und nicht auch das objektive Geschehen bestimmend ist. In dieser Verleugnung des Realitätsgehalts liegt aber selbst eine willkürlich-starre und unreflektierte Verabsolutierung der eigenen Vorstellungsart“" (Zabka 1993, 175). 
Beobachtungen des Homunkulus mit den Worten: „Sei ruhig! Es war nur gedacht“ (V. 7946), so als stünde es nicht allen deutlich vor Augen, was geschehen ist.

Die Diskussion zwischen Thales und Anaxagoras verhält sich zu der vorangegangenen Entstehung des Berges als dessen Nicht-Erklärung. Anders als es die Ausgangssituation des Gesprächs hoffen ließ, hat sich hier nichts entschieden. Die Gegensätze haben sich nicht nur verstärkt, sondern sogar noch vermehrt. Zum Problem der Zeit hat sich die ungeklärte Frage gesellt, welchen Status für die wissenschaftliche Erklärung das sinnliche Datum des seismischen Felsens haben könnte. Das Verhältnis von Anschaulichkeit und Deutung scheint damit von Grund auf gestört, denn die Deutungen überwuchern das Faktum. Die Bergentstehung und das mit ihr verbundene Geschehen verschwinden hinter ihren Diskursivierungen beinahe vollständig. Der Berg liefert nicht, wie es Goethes Ideal war, mit den Phänomenen schon die Theorie, ${ }^{12}$ sondern lässt im Gegenteil zwischen der Anschauung eines Gegenstandes und seiner Erklärung eine Kluft entstehen. Evident scheint nur noch, was jeder dafür hält, ohne dass es für die Rezipienten und Rezipientinnen des Textes die Möglichkeit einer Objektivierung gäbe.

Fassen wir das Gesagte zusammen: Der zweite Teil des Faust erscheint als ein topisch angelegtes Archiv, bei dem Orte und dort anzutreffende Figuren wie Rubriken wirken, die Einträge aus dem dahinterliegenden größeren Archiv der Kultur erlauben, die von den Rezipienten und Rezipientinnen aufgespürt werden können. Dabei geht es nicht um ein wörtliches Zitieren von Texten, sondern um deren theatrale Metamorphose, um die Erzeugung von Charakteren und Handlungen aus dem Geist des Archivs. Dadurch erhält das Werk mehrfache Ebenen der Deutung und es erscheint unter anderem als eine Reflexion auf Entwicklungen in der Wissensgeschichte der damaligen Zeit. Die Archivpoetik erlaubt es Goethe mithin, einen Text als Archiv zu verfassen, der zugleich das Kulturarchiv seiner Zeit beobachtet, kommentiert und zur Erzeugung seiner theatralen Wirklichkeit nutzt. ${ }^{13}$

12 Vgl. LA I/11, 358: „Das Höchste wäre: zu begreifen, daß alles Faktische schon Theorie ist. Die Bläue des Himmels offenbart uns das Grundgesetz der Chromatik. Man suche nur nichts hinter den Phänomenen; sie selbst sind die Lehre.“

13 Dieser Aufsatz entstand dank der freundlichen Aufforderung der Herausgeber, meine früheren Überlegungen zu Goethes Archivpoetik zusammenzufassen (vgl. Schneider 2005). Es handelt sich teilweise um wörtliche Passagen aus meiner Dissertationsschrift, die aber gestrafft und um neue Überlegungen angereichert wurden. 


\section{Literaturverzeichnis}

Asman, Carrie. „Kunstkammer als Kommunikationsspiel. Goethe inszeniert eine Sammlung“. Johann Wolfgang Goethe: Der Sammler und die Seinigen. Hg. Carrie Asman. Dresden: Verl. der Kunst, 1997. 119-177.

Bez, Martin. Goethes, Wilhelm Meisters Wanderjahre؛ Aggregat, Archiv, Archivroman. Berlin, Boston: de Gruyter, 2013.

Bredekamp, Horst. Antikensehnsucht und Maschinenglauben. Die Geschichte der Kunstkammer und die Zukunft der Kunstgeschichte. Berlin: Wagenbach, 1993.

Curtius, Ernst Robert. „Goethes Aktenführung“. Kritische Essays zur europäischen Literatur. 2. erweiterte Aufl. Bern: Francke, 1954. 57-69.

Dotzler, Bernhard J. „Big Number Avalanche \& Weltliteratur. Medienwissenschaftliche Notizen zu Goethes Aktenführung“. Masse und Medium. Verschiebungen in der Ordnung des Wissens und der Ort der Literatur 1800/2000. Hg. Ingrid Münz-Koenen und Wolfgang Schaeffner. Berlin: Akademie, 2002. 3-14.

Flach, Willy. „Goethes literarisches Archiv“. Archivar und Historiker. Studien zur Archiv- und Geschichtswissenschaft. Zum 65. Geburtstag von Heinrich Otto Meisner. Hg. Staatliche Archivverwaltung. Berlin: Rütten und Loening, 1956. 45-71.

Gaier, Ulrich. „Dialektik der Vorstellungsarten als Prinzip in Goethes ,Faust““. Interpreting Goethe's ,Faust' today. Hg. Jane K. Browne, Meredith Lee und Thomas P. Saine, in Zusammenarbeit mit Paul Hernadi und Cyrus Hamlin. Columbia, SC: Camden House, 1994. 158-171.

Gaier, Ulrich. „Kommentar 2“. Johann Wolfgang Goethe: Faust-Dichtungen. Bd. 3/2. Hg. u. kommentiert Ulrich Gaier. Stuttgart: Reclam, 1999.

Goethe, Johann Wolfgang. Die Schriften zur Naturwissenschaft. Vollständige mit Erläuterungen und Kommentaren versehene Ausgabe im Auftrage der Deutschen Akademie der Naturforscher zu Halle (Leopoldina). Begr. Lothar Wolf und Wilhelm Troll. Hg. Dorothea Kuhn und Wolf von Engelhardt. Wien, Köln, Weimar: Böhlau 1947-2011. [LeopoldinaAusgabe, Textbelege unter Sigle LA + Bandnummer]

Goethe, Johann Wolfgang. Faust. Hg. Albrecht Schöne und Hendrik Birus. Frankfurt a. M., Berlin: Dt. Klassiker Verl., 1994 (= Frankfurter Ausgabe 7/1).

Goethe, Johann Wolfgang. Artemis-Gedenkausgabe der Werke, Briefe und Gespräche. Hg. Ernst Beutler. Zürich: Artemis 1948-1961. [Gedenk-Ausgabe, Textbelege unter Sigle $\mathrm{GA}+$ Bandnummer]

Goethe, Johann Wolfgang. Goethes amtliche Schriften. Bd. I. Hg. Willy Flach. Weimar: Böhlau 1950.

Goethe, Johann Wolfgang. Sämtliche Werke. Briefe, Tagebücher und Gespräche. 40 in 45 Bänden in 2 Abteilungen. Hg. Friedmar Apel et al. Frankfurt a. M., Berlin: Dt. Klassiker Verl., 1987-2013. [Frankfurter Ausgabe, Textbelege unter Sigle FA + Bandnummer]

Hölder, Helmut. Geologie und Paläontologie in Texten und ihrer Geschichte. Freiburg, München: Alber, 1960.

Pompe, Hedwig, und Leander Scholz (Hg.). Archivprozesse. Die Kommunikation der Aufbewahrung. Köln: DuMont, 2002.

Pörksen, Uwe. „Raumzeit. Goethes Zeitbegriff aufgrund seiner sprachlichen Darstellung geologischer Ideen und ihrer Visualisierung“. Goethe und die Verzeitlichung der Natur. Hg. Peter Matussek. München: C. H. Beck, 1998. 101-127. 
Schneider, Steffen. Archivpoetik. Die Funktion des Wissens in Goethes ,Faust II‘. Tübingen: Niemeyer, 2005.

Trunz, Erich. „Goethe als Sammler“. Weimarer Goethe-Studien. Weimar: Böhlau, 1980. 7-47. Wagenbreth, Otfried. Geschichte der Geologie in Deutschland. Stuttgart: Thieme Georg Verl., 1999.

Zabka, Thomas. Faust Il. Das Klassische und das Romantische. Goethes, Eingriff in die neueste Literatur. Tübingen: Niemeyer, 1993.

Zedelmaier, Helmut. „Buch, Exzerpt, Zettelschrank, Zettelkasten“. Archivprozesse. Hg. Hedwig Pompe und Leander Scholz. Köln: DuMont, 2002. 38-53. 
